\title{
Is the Use of Intensity-Modulated Radiotherapy Beneficial for Pancreatic Cancer Patients?
}

\author{
Hee Chul Park \\ Department of Radiation Oncology, Samsung Medical Center, Sungkyunkwan University School of Medicine, Seoul, Korea
}

See "A Comparison of Gastrointestinal Toxicities between Intensity-Modulated Radiotherapy and Three-Dimensional Conformal Radiotherapy for Pancreatic Cancer" by Kyong Joo Lee, et al. on page 303-309, Vol. 10. No. 2, 2016

Intensity-modulated radiotherapy (IMRT) represents one of the major technological innovations in the modern radiotherapy era. It is an advanced form of three-dimensional conformal radiotherapy (3-D CRT) that applies nonuniform beam intensity with computer-aided inverse planning to achieve superior dose distribution. ${ }^{1}$ The biggest advantage of IMRT is its ability to produce a much greater conformity of the dose distribution than achievable with conventional 3-D CRT using uniform beam intensities. The ability to manipulate the dose distribution can be utilized to create sharp dose fall-off near the boundaries of the tumor area and critical normal organs. This means that the proportion of critical organs receiving a high dose of radiation could be greatly reduced. This may allow for an increase in the dose delivered to the tumor area, a reduction in the radiation dose delivered to critical organs, or both, which may potentially lead to improved clinical outcomes.

When a major innovation is introduced, many new issues that may challenge existing technologies or clinical knowledge are encountered. The improved dose distribution quality resulting from the use of IMRT is due to the trade-off between high dose conformity and the increased radiation exposure of normal tissue. As a result, when sparing organs at risk, the dose is redistributed to other normal tissues, possibly causing unintended tissue toxicity. ${ }^{2,3}$ Therefore, clinical trial evidence is needed to support the theoretical dosimetric superiority of IMRT for its routine application in specific clinical scenarios.

The recent development of IMRT and improvements in the image-guided delivery of radiotherapy have provided considerable advances in the adoption of radiotherapy for the management of pancreatic cancer. ${ }^{3}$ In this issue of Gut and Liver, Lee et al. ${ }^{4}$ demonstrate that IMRT is associated with significantly fewer gastroduodenal injuries among pancreatic cancer patients treated with concurrent chemoradiotherapy. These authors evaluated the acute toxicities of dyspepsia, nausea, vomiting, and diarrhea. Additionally, preplanned evaluations with gastroduodenoscopies were conducted to evaluate radiation-induced gastroduodenal injuries including telangiectasia, erythema, ulcers, and scar formation. In the IMRT-based chemoradiotherapy arm, the development of grade 1 to 2 ulcers was significantly reduced. Based on the superiority of the endoscopic results, the authors conjectured that IMRT resulted in significantly fewer gastroduodenal injuries among patients treated with concurrent chemoradiotherapy for pancreatic cancer. Several reports have been published on the use of IMRT for pancreatic cancer, and most of the studies were single-arm prospective studies that mainly adopted a 5-fluorouracil-based chemotherapy regimen. ${ }^{5-11}$ To my knowledge, the study by Lee et al. ${ }^{4}$ is the first to directly compare 3-D CRT and IMRT for the management of pancreatic cancer.

However, due to the nature of their cohort trial, the proportion of locally advanced cases was increased in the 3-D CRT arm, as the authors noted themselves. Their reduced toxicity profiles are not clearly supported by an improvement in the dose volume statistics, which were evaluated using IMRT for comparison. To arrive at a firm conclusion that IMRT is better for the management of pancreatic cancer, further well-balanced, randomized trial results are required.

IMRT is a modality that is sensitive to the motion of the target and the surrounding normal organs because inverse planning optimization of the individual beam intensity is performed

Correspondence to: Hee Chul Park

Department of Radiation Oncology, Samsung Medical Center, Sungkyunkwan University School of Medicine, 81 Irwon-ro, Gangnam-gu, Seoul 06351, Korea

Tel: +82-2-3410-2612, Fax: +82-2-3410-2619, E-mail: rophc@skku.edu pISSN 1976-2283 eISSN 2005-1212 http://dx.doi.org/10.5009/gnl16014

@) This is an Open Access article distributed under the terms of the Creative Commons Attribution Non-Commercial License (http://creativecommons.org/licenses/by-nc/4.0) which permits unrestricted non-commercial use, distribution, and reproduction in any medium, provided the original work is properly cited. 
based on the fixed computed tomography (CT) image set during simulation. The shape and position of the pancreas and the surrounding normal organs are significantly affected by respiratory motion and/or the motility of the gastrointestinal tract. To overcome the potential changes in the ideally planned distribution of the IMRT dose, a thorough consideration of the motionrelated issues during simulation, planning of the procedure and daily radiation delivery are necessary. Recently, image-guided technologies have emerged with advances in intensity modulation; this potential risk while delivering IMRT to a moving target will be overcome rapidly because the study of Lee et al. ${ }^{4}$ utilized in-room megavoltage CT guidance and abdominal compression to minimize the effect of organ motion.

Is the use of IMRT beneficial for pancreatic cancer treatment? IMRT provides better conformity with the tumor target and reduces the dose delivered to organs at risk. This may translate into better local control and reduced acute and late toxicities to critical organs. However, for a firm conclusion, further studies are required.

\section{CONFLICTS OF INTEREST}

No potential conflict of interest relevant to this article was reported.

\section{REFERENCES}

1. Intensity Modulated Radiation Therapy Collaborative Working Group. Intensity-modulated radiotherapy: current status and issues of interest. Int J Radiat Oncol Biol Phys 2001;51:880-914.

2. Reese AS, Das SK, Kirkpatrick JP, Marks LB. Quantifying the dosimetric trade-offs when using intensity-modulated radiotherapy to treat concave targets containing normal tissues. Int J Radiat Oncol Biol Phys 2009;73:585-593.
3. Reese AS, Lu W, Regine WF. Utilization of intensity-modulated radiation therapy and image-guided radiation therapy in pancreatic cancer: is it beneficial? Semin Radiat Oncol 2014;24:132-139.

4. Lee KJ, Yoon HI, Chung MJ, et al. A comparison of gastrointestinal toxicities between intensity-modulated radiotherapy and three-dimensional conformal radiotherapy for pancreatic cancer. Gut Liver 2016;10:303-309.

5. Milano MT, Chmura SJ, Garofalo MC, et al. Intensity-modulated radiotherapy in treatment of pancreatic and bile duct malignancies: toxicity and clinical outcome. Int J Radiat Oncol Biol Phys 2004;59:445-453.

6. Fuss M, Wong A, Fuller CD, Salter BJ, Fuss C, Thomas CR. Imageguided intensity-modulated radiotherapy for pancreatic carcinoma. Gastrointest Cancer Res 2007;1:2-11.

7. Yovino S, Poppe M, Jabbour S, et al. Intensity-modulated radiation therapy significantly improves acute gastrointestinal toxicity in pancreatic and ampullary cancers. Int J Radiat Oncol Biol Phys 2011;79:158-162.

8. Abelson JA, Murphy JD, Minn AY, et al. Intensity-modulated radiotherapy for pancreatic adenocarcinoma. Int J Radiat Oncol Biol Phys 2012;82:e595-e601.

9. Ben-Josef E, Schipper M, Francis IR, et al. A phase I/II trial of intensity modulated radiation (IMRT) dose escalation with concurrent fixed-dose rate gemcitabine (FDR-G) in patients with unresectable pancreatic cancer. Int J Radiat Oncol Biol Phys 2012;84:1166-1171.

10. Son SH, Song JH, Choi BO, et al. The technical feasibility of an image-guided intensity-modulated radiotherapy (IG-IMRT) to perform a hypofractionated schedule in terms of toxicity and local control for patients with locally advanced or recurrent pancreatic cancer. Radiat Oncol 2012;7:203.

11. Tunceroglu A, Park JH, Balasubramanian S, et al. Dose-painted intensity modulated radiation therapy improves local control for locally advanced pancreas cancer. ISRN Oncol 2012;2012:572342. 